\title{
CT imaging changes of corona virus disease 2019(COVID-19): a multi-center study in Southwest China
}

\author{
Xiaoming $\mathrm{Li}^{1 \dagger}$, Wenbing Zeng ${ }^{2 \dagger}$, Xiang $\mathrm{Li}^{2}$, Haonan $\mathrm{Chen}^{2}$, Linping Shi ${ }^{3}$, Xinghui $\mathrm{Li}^{4}$, Hongnian Xiang ${ }^{5}$, \\ Yang $\mathrm{CaO}^{6}$, Hui Chen ${ }^{1}$, Chen Liu ${ }^{1 *}$ and Jian Wang ${ }^{1 *}$
}

\begin{abstract}
Background: Since the first case of a coronavirus disease 2019 (COVID-19) infection pneumonia was detected in Wuhan, China, a series of confirmed cases of the COVID-19 were found in Southwest China. The aim of this study was to describe the imaging manifestations of hospitalized patients with confirmed COVID-19 infection in southwest China.
\end{abstract}

Methods: In this retrospective study, data were collected from 131 patients with confirmed coronavirus disease 2019 (COVID-19) from 3 Chinese hospitals. Their common clinical manifestations, as well as characteristics and evolvement features of chest CT images, were analyzed.

Results: A total of 100 (76\%) patients had a history of close contact with people living in Wuhan, Hubei. The clinical manifestations of COVID-19 included cough, fever. Most of the lesions identified in chest CT images were multiple lesions of bilateral lungs, lesions were more localized in the peripheral lung, 109 (83\%) patients had more than two lobes involved, 20 (15\%) patients presented with patchy ground glass opacities, patchy ground glass opacities and consolidation of lesions co-existing in 61 (47\%) cases. Complications such as pleural thickening, hydrothorax, pericardial effusion, and enlarged mediastinal lymph nodes were detected but only in rare cases. For the follow-up chest CT examinations (91 cases), We found 66 (73\%) cases changed very quickly, with an average of 3.5 days, 25 cases (27\%) presented absorbed lesions, progression was observed in 41 cases (46\%), 25 (27\%) cases showed no significant changes.

Conclusion: Chest CT plays an important role in diagnosing COVID-19. The imaging pattern of multifocal peripheral ground glass or mixed consolidation is highly suspicious of COVID-19, that can quickly change over a short period of time.

Keywords: Coronavirus, The chest, Computed tomography, Pneumonia, Evolvement

\section{Background}

An epidemic of the 2019 novel coronavirus (2019-nCoV) erupted in Wuhan, Hubei Province of China at the end of

\footnotetext{
*Correspondence: liuchen@aifmri.com; wangjian_811@yahoo.com ${ }^{\dagger}$ Xiaoming Li and Wenbing Zeng equal contributions to this work 1 Department of Radiology, First Affiliated Hospital to Army Medical University, Chongqing, China

Full list of author information is available at the end of the article
}

December 2019, and has quickly spread across the country, including overseas. As of February 23, 2020, 77,041 confirmed cases have been reported in China, with a cumulative death toll of 2445 . As a newly identified member of the coronavirus-beta subfamily, the virus shares more than $85 \%$ homology with the bat severe acute respiratory syndrome (SARS)-like coronavirus (bat-SLCoVZC45), belonging to the same class as the SARS-CoV found in 2003 and the Middle East respiratory syndrome

c) The Author(s) 2020. This article is licensed under a Creative Commons Attribution 4.0 International License, which permits use, sharing, adaptation, distribution and reproduction in any medium or format, as long as you give appropriate credit to the original author(s) and the source, provide a link to the Creative Commons licence, and indicate if changes were made. The images or other third party material in this article are included in the article's Creative Commons licence, unless indicated otherwise in a credit line to the material. If material is not included in the article's Creative Commons licence and your intended use is not permitted by statutory regulation or exceeds the permitted use, you will need to obtain permission directly from the copyright holder. To view a copy of this licence, visit http://creativeco mmons.org/licenses/by/4.0/. The Creative Commons Public Domain Dedication waiver (http://creativecommons.org/publicdomain/ zero/1.0/) applies to the data made available in this article, unless otherwise stated in a credit line to the data. 
virus (MERS-CoV) found in 2012. Whole-genome sequencing and genetic analysis of the strain has demonstrated that the virus is a different branch from SARS$\mathrm{CoV}$ and MERS-CoV [1]. Currently, the epidemic that originated in Wuhan is ongoing. On February 11, 2020, the International Committee on Taxonomy of Virus (ICTV) officially named 2019-nCoV as "severe acute respiratory syndrome coronavirus 2" (SARS-CoV-2) [2]. Furthermore, the World Health Organization (WHO) has named the disease caused by infection with this virus as Coronavirus Disease 2019 (COVID-19). Generally, the incubation period of COVID-19 ranges from 1 to 14 days, with most people developing symptoms between 3-7 days; however, the longest incubation period can reach 24 days [3]. The clinical severity of COVID-19 varies greatly, from asymptomatic to death.

Currently, a positive result in nucleic acid testing (NAT) using reverse-transcriptase polymerase-chainrection (RT-PCR) technology is the gold standard for diagnosing COVID-19. The assay has high specificity, but low sensitivity. Hence, chest $\mathrm{CT}$ has become a critical diagnostic tool for COVID-19, used in close combination with clinical manifestations and epidemiological evidence for disease confirmation. However, discrepancies between the results of NAT and imaging features have been reported in the literature. For example, the CT images of some patients showed apparent lesions in the lungs, yet NAT repeatedly showed negative results until eventually turning positive [4]. Recently, Fang et al. observed that in diagnosing COVID-19, the sensitivity of CT (98\%) was significantly higher than that of RT-PCR (71\%) [5]. China's Diagnosis and Treatment Plan for Novel Coronavirus-infected Pneumonia (5th Trial Edition) has established CT imaging as the clinical diagnosis in Hubei Province. Therefore, there is an urgent need for further studies to provide data and evidence on the manifestations of COVID-19 in chest CT imaging.

In this study, the clinical manifestations, epidemiology, laboratory test results, and chest CT findings of 131 confirmed COVID-19 patients were summarized and investigated, to improve the understanding of the disease, thereby achieving effective control of the epidemic through early diagnosis, prompt treatment, and quarantine, beneficial to the timely implementation of measures for monitoring public health.

\section{Materials and methods}

\section{General information}

Relevant data of COVID-19 patients admitted to 3 hospitals during the period from December 28, 2019 to February 10, 2020 were retrospectively collected. The inclusion criteria were as follows: (1) patient tested positive for NAT using RT-PCR; (2) patient received the first chest CT examination after admission to hospital. A total of 131 patients were finally enrolled in the study (Table 1), including 63 males and 68 females, aged between 20-90 years. The average age of all the patients was $47 \pm 15$ years, with males averaging at 45 years and females at 49 years. The time interval between disease onset and admission to hospital ranged between 1 and 19 days, with an average of 4.6 days. The results of a series of laboratory tests were recorded, including the white blood cell count (WBC) (normal range 3.5-9.5 $\times 10^{9} / \mathrm{L}$ ), neutrophils (normal range $1.8-6.3 \times 10^{9} / \mathrm{L}$ ) and lymphocytes (normal range 1.1-3.2 $\times 10^{9} / \mathrm{L}$ ), procalcitonin (normal range $0-0.07 \mathrm{ng} / \mathrm{ml}$ ), and C-reactive protein (CRP) (normal range $0-10 \mathrm{mg} / \mathrm{L}$ ).

Table 1 Baseline characteristics of the overall study population

\begin{tabular}{ll}
\hline Variable & Patients ( $\mathbf{n = 1 3 1 )}$ \\
\hline Age (years) & $20-90$ \\
Sex & \\
M & $63(48 \%)$ \\
F & $68(52 \%)$ \\
Exposure history & \\
Close contact & $100(76 \%)$ \\
Uncertainty & $31(24 \%)$ \\
Clinical symptoms & \\
Fever & $85(65 \%)$ \\
Cough & $85(65 \%)$ \\
Feeble & $13(10 \%)$ \\
Shortness of breath & $5(4 \%)$ \\
Muscle ache & $2(2 \%)$ \\
Diarrhoea & $1(1 \%)$ \\
Blood biochemistry & \\
Leucocytes $\left(\times 10^{9}\right.$ per L) & \\
Decreased & \\
Normal & \\
Increased & $11(8 \%)$ \\
Neutrophils $\left(\times 10^{9}\right.$ per L) & $111(85 \%)$ \\
Decreased & $9(7 \%)$ \\
Normal &
\end{tabular}




\section{Examination methods}

All patients were required to wear masks and walk to the CT scan facility using a hallway exclusively for outpatients with fever. Disposable bed sheets were used during each examination. CT scans were performed using the SOMATOM Definition AS + 64 row spiral CT (Siemens, Germany) (First Affiliated Hospital to Army Medical University and Affiliated Hospital of North Sichuan Medical College), and Emotion 16 row spiral CT (Siemens, Germany) (Chongqing Three Gorges Central Hospital) Scan parameter settings were as follows: tube voltage at default machine settings, with automatic tube current, layer thickness ranged at $2-5 \mathrm{~mm}$, and layer spacing ranged at $2-5 \mathrm{~mm}$. The scan covered the area from the thoracic inlet to the costophrenic angle. As patients with relatively severe symptoms found it difficult to hold their breath, the scan was performed in a reverse direction from the costophrenic angle to the thoracic inlet. The lung window was reconstructed with a high-resolution algorithm at $1.0 \mathrm{~mm}$. Sagittal and coronal reconstructions were performed using post-processing workstations. The machine and the room were thoroughly disinfected after the examination of each patient.

\section{Image analysis}

The chest CT of each patient was independently reviewed by two radiologists with 5 and 10 years of experience in CT diagnostics. In case of any discrepancy, a consensus was reached through discussion. The images were analyzed for the following aspects: (1) presence of groundglass opacities: defined by increase in lung density but without covering the pulmonary blood vessels and bronchial walls; (2) presence of lung consolidation: defined by higher density than ground-glass opacities and blurred margins of pulmonary blood vessels and bronchial tubes; (3) presence of nodular/cord-like shadows; (4) interlobular septal thickening, thickening of vascular, and air bronchogram signs inside the lesions; (5) number of lesions (recorded as 1, 2, 3, and $>3$; numbers $>3$ were recorded as multiple without the specific number); (6) lesion site (left and right lobe, center of the lung, near the hilum/peripheral: far from the hilum); (7) complications other diseases such as pleural thickening, hydrothorax, pericardial effusion, or lymphadenopathy (short diameter $\geq 1 \mathrm{~cm}$ ); (8) disease progression: defined by comparing the scope, quantity, and density of lesions detected in two chest CT scans. Obviously fusion of lesions, new lesions, and (or) increased lung density was considered progression. Conversely, obviously reduced lesion size, number, and (or) density was considered absorption. If no significant difference in the lesions between the two CT examinations was observed, the patient's condition was considered stable.

\section{Results}

\section{Findings of first chest CT scan}

With the 6 cases were negative in the chest CT, 125 of 131 patients presented abnormal. Bilateral involvement was identified in 104 cases (79\%), 14 cases $(11 \%)$ showed lesions in the right lung, while 7 cases (5\%) reported lesions in the left lung. 16 cases $(12 \%)$ involved one lobe, 12 cases (9\%) involved two lobe, 13 cases (10\%) involved three lobe, 16 cases (12\%) involved four lobe, and 68 cases (52\%) involved five lobe; The lesions were peripherally distributed in 100 cases (76\%), occurred in both peripheral and central locations in 24 cases $(18 \%)$, and in the center in 1 case (1\%). Multiple lesions $(>3)$ were detected in 115 cases (87\%), 1 case (1\%) presented 3 lesions, 3 cases $(2 \%)$ showed 2 lesions, and 6 cases $(5 \%)$ reported 1 lesion.

Chest CT imaging manifestations of the 125 positive cases included 106 cases (81\%) with patchy groundglass opacities, 91 cases $(69 \%)$ with patchy consolidations, 40 cases (31\%) with nodules, and 94 cases $(72 \%)$ with two or more forms of lesions co-existing (Fig. 1a-d). The lesion margins were clear in 60 patients (46\%) but blurry in the remaining 65 cases (50\%). 68 cases $(52 \%)$ demonstrated interlobular septal thickening (8 cases presented typical "crazy paving" pattern). Thickening of vascular, air bronchogram signs, or fibrous foci (Table 2). In 1 case, a consolidation with cavity in the right lower lobe. In 3 cases, "reverse halo signs" were detected, and the initial chest CT of 1 case presented nodules, the "reversed halo sign" appeared in the 2 days follow up reexamination in the left lower lobe.

\section{Findings of second chest $\mathrm{CT}$ scan}

Among the 131 patients, 91 underwent a second chest CT scan (Table 3). The time interval between the two scans ranged from 2 to 7 days, with an average of 3.5 days. In the follow-up, 25 patients (27\%) presented absorbed lesions (Fig. 2), The chest CT images of another 25 patients (27\%) showed no significant changes (Fig. 3). Disease progression was observed in 41 cases $(46 \%)$ (Fig. 4), including the 3 patients with negative results in the first scan and new lesions in the second scan performed 2, 4 and 5 days after the first scan (Fig. 5).

\section{Discussions}

SARS-CoV-2 is a single-stranded RNA virus that can be transmitted from human to human; comparable to the transmission of SARS and MERS, SARS-CoV-2 is also transmitted primarily through respiratory droplets and contact $[6,7]$. In this study, we had marginally more female subjects than male subjects, which could be related to the fact that the average age of men was lower than that of women. The major clinical symptoms 


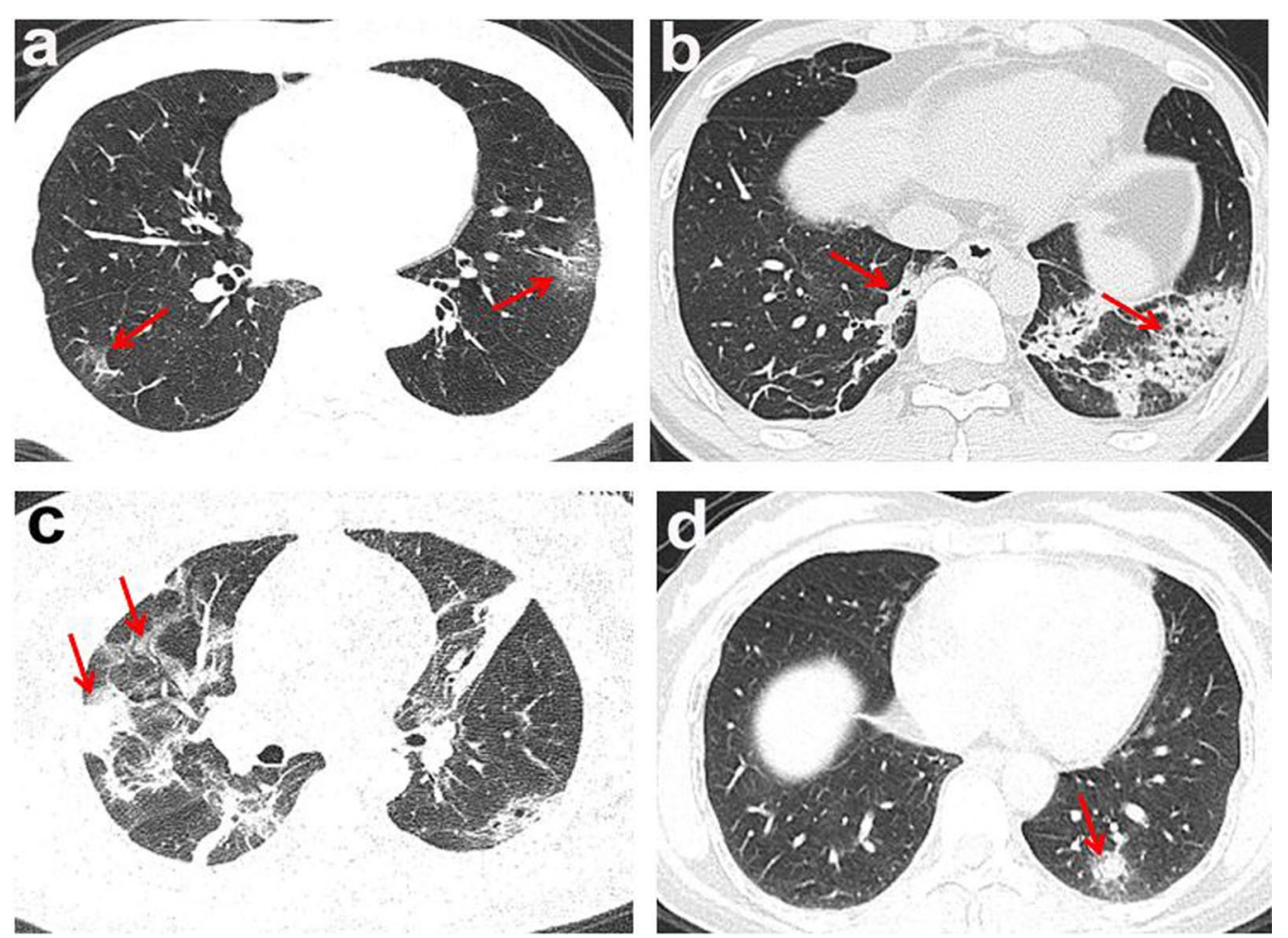

Fig. 1 The lesion of multiple morphologic manifestations. The red arrows and boxes indicated the abnormalities. a Ground glass opacities; $\mathbf{b}$ consolidation; c consolidation with ground glass opacities; $\mathbf{d}$ solid nodule (red arrow)

Table 2 Innitial chest CT findings of 125 patients

\begin{tabular}{ll}
\hline Morphology & Patients $(\mathbf{n}=\mathbf{1 3 1})$ \\
\hline Ground-glass opacities* & $20(15 \%)$ \\
Consolidation* $^{*}$ & $4(3 \%)$ \\
Nodule* $^{*}$ & $7(5 \%)$ \\
Ground-glass opacities and consolidation & $61(47 \%)$ \\
Ground-glass opacities and nodule & $7(5 \%)$ \\
Consolidation and nodule & $8(6 \%)$ \\
Both of all & $18(14 \%)$ \\
With others & \\
Interlobular septal thickening & $68(52 \%)$ \\
Vascular enlargement & $84(64 \%)$ \\
Air bronchogram & $75(57 \%)$ \\
Fibrosis & $43(33 \%)$ \\
Pleural thickening & $31(24 \%)$ \\
Hydrothorax & $3(2 \%)$ \\
Lymph node enlargement & $17(13 \%)$ \\
\hline
\end{tabular}

*Without the other two morphology

observed included fever (65\%), cough (65\%), consistent with previous reports [3]. Notably, $76 \%$ of the patients had a history of close contact with people living in Wuhan. Although the contact history of the other $24 \%$ was unclear, these patients worked in crowded environments where numerous people gathered (such as restaurants and hotels).

In this group of patients, the $\mathrm{WBC}$ and neutrophil counts and the procalcitonin levels were normal in most cases, but the CRP was elevated, consistent with the report by Chen et al. [8]. Lymphocyte count was decreased in 74 patients $(57 \%)$, and the average age were $51 \pm 16$ years; 57 patients (43\%) presented normal lymphocyte counts, and the average age were $42 \pm 11$ years $(P=0.002)$. The results indicated that with increased patients' age the immune cells were more susceptible to damage by the virus, and hence their immunity was weakened [9].

Initial chest $\mathrm{CT}$ scan failed to reveal any lesions in 6 of the 131 patients, while the CT findings of the remaining 125 cases showed that the majority of lesions (79\%) involved bilateral lungs, $88 \%$ of the lesions involved two or more lobes simultaneously, and nearly $76 \%$ of the lesions were distributed in the periphery of the lung. The lesions were not confined to certain segments of lung lobes, possibly due to the small size of the virions that tend to deposit on the lobules in the periphery of the lung, thereby causing damage to the alveolar epithelium and affecting multiple adjacent lobules [10]. 
Table 3 The follow-up results of chest CT in 91 patients

\begin{tabular}{|c|c|c|}
\hline Follow-up results(n) & Innitial chest $\mathrm{CT}$ findings & Patients $(n=91)$ \\
\hline \multirow[t]{6}{*}{ Absorb (25) } & Ground-glass opacities & $2(8 \%)$ \\
\hline & Consolidation & $1(4 \%)$ \\
\hline & Ground-glass opacities and consolidation & $17(68 \%)$ \\
\hline & Ground-glass opacities and nodule & $1(4 \%)$ \\
\hline & Consolidation and nodule & $1(4 \%)$ \\
\hline & Both of all & $3(12 \%)$ \\
\hline \multirow[t]{5}{*}{ Stable (25) } & Ground-glass opacities & $3(12 \%)$ \\
\hline & Nodule & $2(8 \%)$ \\
\hline & Ground-glass opacities and consolidation & $13(52 \%)$ \\
\hline & Consolidation and nodule & $4(6 \%)$ \\
\hline & Both of all & $3(12 \%)$ \\
\hline \multirow[t]{7}{*}{ Advance (41) } & Ground-glass opacities & $9(22 \%)$ \\
\hline & Consolidation & $2(5 \%)$ \\
\hline & Nodule & $4(10 \%)$ \\
\hline & Ground-glass opacities and consolidation & $14(34 \%)$ \\
\hline & Ground-glass opacities and nodule & $5(2 \%)$ \\
\hline & Both of all & $4(10 \%)$ \\
\hline & $(-)^{*}$ & $3(7 \%)$ \\
\hline
\end{tabular}

*The first chest CT examination was negative

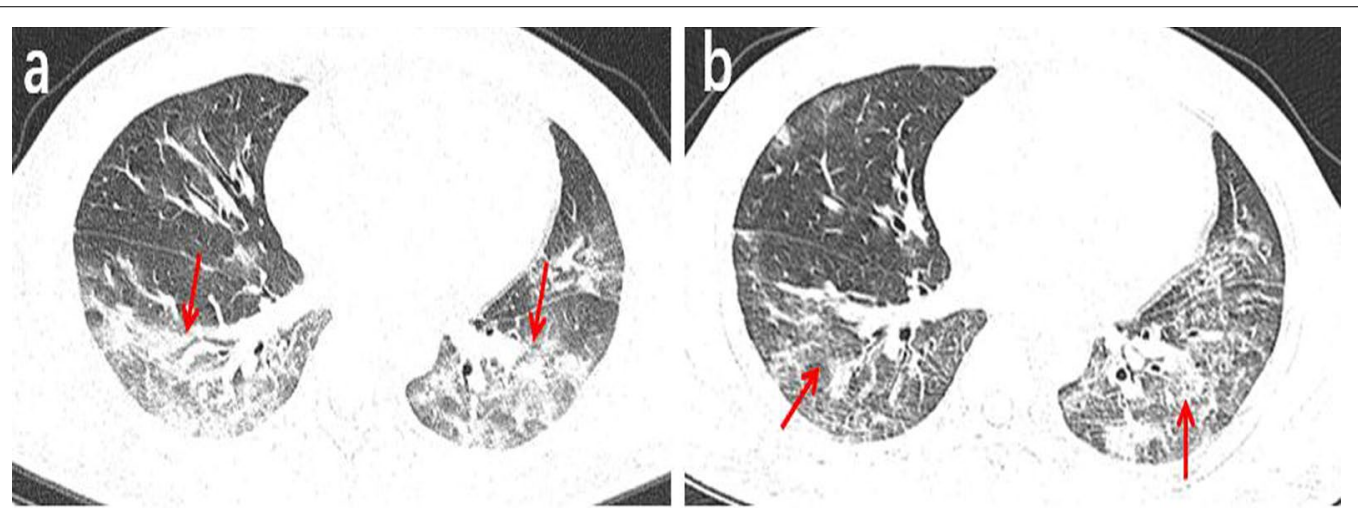

Fig. 2 A 38-year-old male working at a hotel presented with a cough, fever, and fatigue for 10 days. a The first axial-view chest CT shows diffused, mixed shadows of ground-glass opacities and consolidations (red arrows) with blurred margins. b In the second axial-view chest CT scan conducted 2 days after the first one, the lesion density is significantly reduced and the scope of lesions is narrowed (red arrows)

Common chest CT manifestations of COVID-19 included: (1) patchy ground-glass opacities with clear margins and visible interlobular septal thickening inside the lesions were observed in 106 cases (81\%), some cases appeared a typical " crazy paving pattern", and visible vascular thickening. This can be explained by the virusinduced diffuse alveolar wall injury, vascular congestion, and alveolar septal inflammation [11]. (2) Increased lesion density, along with disease progression manifested as patchy consolidations, was detected in 91 cases (69\%). These pathological changes could be attributed to alveolar wall collapse, causing the replacement of alveoli by exudates or products of other diseases (such as cells and epithelium) [12]. Recently, the autopsy of the first COVID-19 patient who died in China revealed significant shedding of the alveolar epithelium and the formation of the pulmonary hyaline membrane [13], consistent with the clinical symptoms of COVID-19, i.e. cough without substantial sputum. (3) Mixed lesions of ground-glass opacities and consolidations was observed in 79 patients (61\%), with or without nodule. Diffused, bilateral pulmonary lesions similar to "white lungs" was observed in 

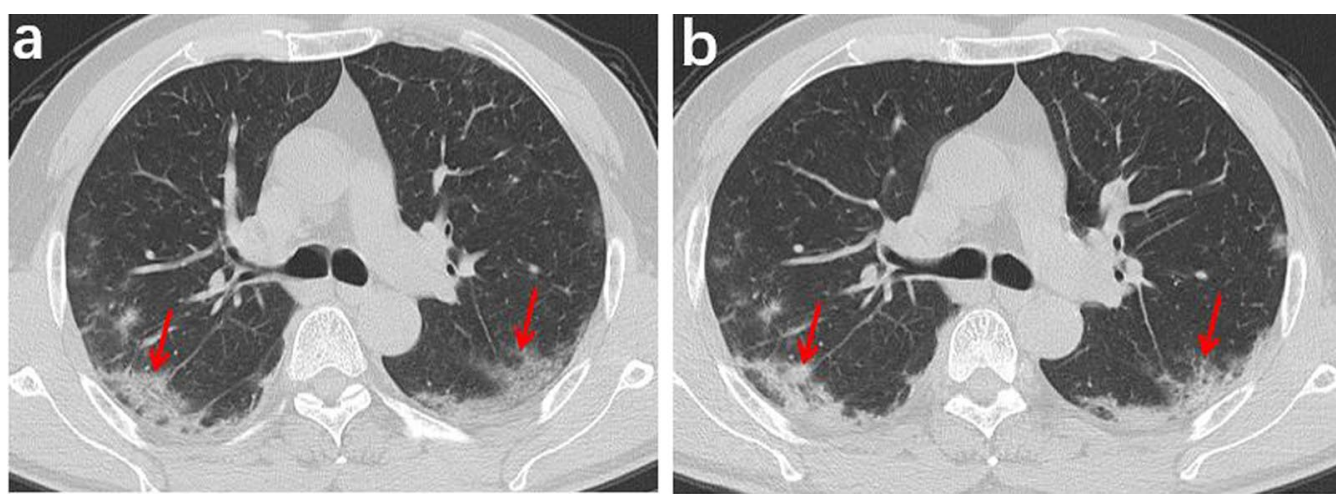

Fig. 3 A 55-year-old male who had close contact with people in Wuhan presented fever for 8 day. a The initial axial chest CT shows mixed shadows of ground-glass opacities and consolidations with peripherally distributed (red arrows). b The follow-up axial-view chest CT shows no significant changes after 4 days later (red arrows)

\section{a}

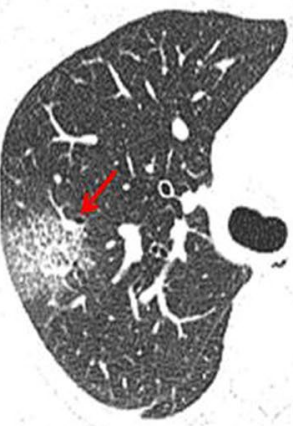

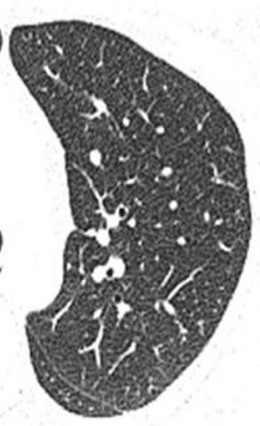

T

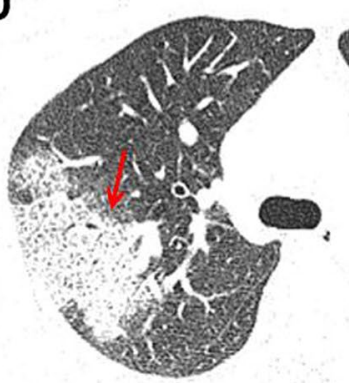

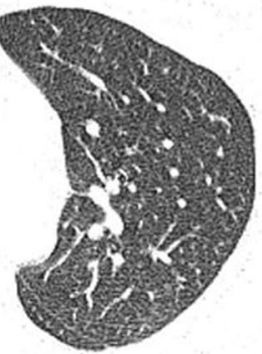

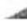

Fig. 4 A 46-year-old female who had close contact with people in Wuhan presented fever for 1 day. a Axial-view chest CT shows ground-glass opacities in the upper right lobe with clear margins and visible interlobular septal thickening inside, forming the "crazy paving sign" (red arrow). b Follow-up axial-view chest CT scan 3 days after the first one shows that the scope of lesions increases significantly, and the lesion density also increases, along with significant thickening of the intralobular and interlobular septa (red arrow)
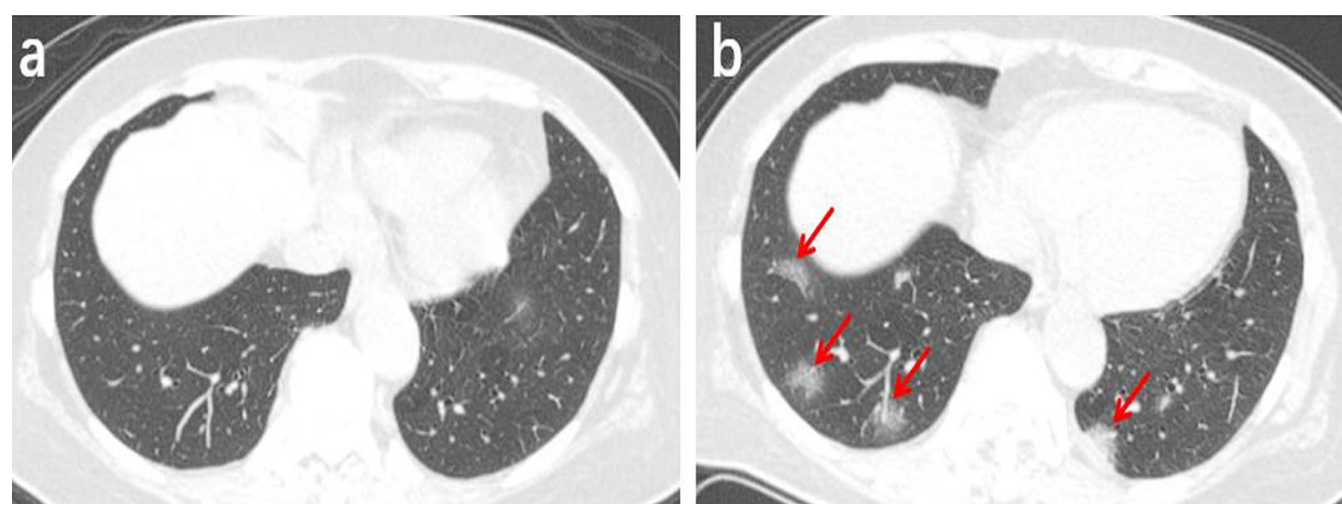

Fig. 5 A 64-year-old female Wuhan resident presented with fever and coughs for 3 days. a The first axial-view chest CT scan shows no lesions. $\mathbf{b}$ The second axial-view chest CT scan conducted 4 days after the first CT shows the presence of multiple ground-glass opacities in the lower lobe, located below the pleura with clear margins. Thickened vascular are visible inside the lesions in the lower right lobe (red arrows) 
10 patients. In these cases, consolidations constituted most of the lesions, with a small percentage demonstrating complicated pulmonary fibrotic foci, which might be related to the fact that the lesions were in the repairing phase [14].

Uncommon chest CT manifestations of COVID-19 included: (1) nodular lesions in 40 patients (31\%). Only 7 of them had simple nodular lesions, whereas the rest had mixed nodular lesions with either ground-glass opacities or consolidations, and the other two signs are the main manifestations. (2) CT showing low central but high peripheral lesion density, or the so-called "reversed halo sign", in the first CT scan of 1 patient. In another patient, the first CT scan showed nodular lesions, but the "reversed halo sign" emerged in the follow-up CT scan 2 days after. The pathological mechanism remains unknown, but this is not a specific imaging characteristic of COVID-19. Other diseases such as organizing pneumonia, cryptococcosis, and tuberculosis can also present the "reversed halo sign" with different pathological mechanisms [15]. (3) A small number of cases showed thickening of adjacent pleura, hydrothorax, pericardial effusion, and enlarged mediastinal lymph nodes, consistent with previous reports [16]. (4) In 1 case, a consolidation with cavity, it might be related to the bronchial discharge of necrotic material of the lesion. This was an extremely rare phenomenon.

Although the time interval between the two CT examinations for the 91 evaluated cases was short (average 3.5 days), but 66 (73\%) patient's chest CT results demonstrated rapid changes, appear significant progress or absorption. The other 25 (27\%) cases was stable. From Table 3, it should note that mixed ground glass and consolidation with or without nodules in the first CT scan. These patients can be progressed, absorbed or stable. However, initial pure patchy ground-glass density imaging indicates a disease aggravated. The risk factors of disease progression are ambiguous. Recently, Liu [17] and Guo [18] have both reported that low lymphocyte counts and hypertension are predictors for worsening conditions, which could be related to the damaged immune system.

The imaging manifestations of COVID-19 are markedly similar to those of SARS and MERS $[19,20]$. (1) SARS often presents as single lesion involving unilateral lung, and septal thickening is evident after the 2nd week [21]. (2) MERS is associated with high mortality. It's difficult to distinguish the imaging from COVID-19, but pleural effusion and pneumothorax are more common in died patients cause by MERS [22]. (3) Early manifestation of influenza virus is primarily tracheobronchitis which presented as nodules or patchy shadows around the bronchial. Centrilobular nodules, pneumatocele formation and lymphadenopathy are often seen in influenza A [23].
To some extent, combined with epidemiology and chest CT can distinguish COVID-19 from other viruses. (4) For mycoplasma pneumonia, the major manifestations include reduced light transmittance of the lung lobes, thickened vascular, thickened bronchial walls, and visible peripheral nodules, presenting the "tree-in-bud" pattern. The disease is commonly complicated by pleural effusion and enlarged mediastinal and hilar lymph nodes [24]. (5) Bacterial pneumonia is often community-acquired or hospital-acquired, with increased WBCs and neutrophils and imaging often showing patchy, nodular, or consolidation shadows distributed along the bronchi or lung segments, which can be distinctly recognized [25]. (6) In cases of cryptococcus infections, lesions are mostly located under the pleura with single or multiple consolidations and nodular shadows, and the disease progresses slowly [26].

The mechanisms during the lesion mainly with ground glass no specific antiviral drugs against progression remain unclear in early stage. We speculate that antiviral drugs may only work at certain times. This phenomenon requires further antivirus study on early intervention. The radiomics will help identify microscopic features of ground glass in early stage. This work may be conducive to differentiate COVID-19 from other viral pneumonia and guide antiviral treatment evaluation.

Our study was limited in the following aspects: (1) Although we summarize the imaging feature of COVID19, quantitative assessments of different pneumonia lesions need to be done in the future. (2) An in-depth study on whether there is a correlation between the disease course and its imaging manifestations was not conducted due to lack of prognostic data. (3) Not all relevant clinical information was collected. For some patients, the lab tests were incomplete, e.g. liver functions and blood coagulation were not examined.

\section{Conclusion}

In summary, the chest CT manifestations of COVID-19 often presented patchy ground-glass opacities or mixed ground-glass opacities and consolidation, involving the periphery of bilateral lungs, that can quickly change over a short period of time. COVID-19 is highly suspected if the patient also has reduced lymphocytes along with epidemiological evidence. Furthermore, it should be noted that some patients with normal chest CT imaging could demonstrate a positive NAT result.

\section{Abbreviations}

COVID-19: Corona Virus Disease 2019; 2019-nCov: 2019 Novel coronavirus; SARS: Severe acute respiratory syndrome; MERS: Middle East respiratory syndrome; SARS-CoV-2: Severe acute respiratory syndrome coronavirus 2; WHO: World Health Organization; NAT: Nucleic acid testing; RT-PCR: Reversetranscriptase polymerase-chain-reaction. 


\section{Acknowledgements}

We thank to the data provided by the Hubei Provincial Corps Hospital Chinese People's Armed Police Forces, Affiliated Hospital of North Sichuan Medical College, and Chongqing Three Gorges Central Hospital.

\section{Authors' contributions}

LX and ZW designed the study; LX was a major contributor in writing the manuscript; LC and WJ reviewed manuscript; LXiang, CH and LXing collected the imaging; SL and XH collected the clinical data; CHui analyzed format. All authors read and approved the final manuscript.

\section{Fundings}

This study was supported by the research about Chongqing Key technology and application demonstration of medical imaging depth intelligent diagnostic platform (cstc2018jszX-cyztzxX0017), Nursery Talents of Army Medical University (2017MPRC-07), Military medical innovation ability improvement plan of medical staff in the First Affiliated Hospital to Army Medical University (SWH2018QNWQ-04) and National Key Research and Development Project (2016YFC0107101)

\section{Availability of data and materials}

All data generated or analyzed during this study are included in this article.

\section{Ethics approval/consent to participate}

The study was conducted in accordance with the principles of the Declaration of Helsinki. This retrospective was approved by the Ethics Committee.

\section{Consent for publication}

Not applicable.

\section{Competing interests}

The authors declare that they have no competing interests.

\section{Author details}

${ }^{1}$ Department of Radiology, First Affiliated Hospital to Army Medical University, Chongqing, China. ${ }^{2}$ Department of Radiology, Three Gorges Central Hospital, Chongqing, China. ${ }^{3}$ Department of Radiology, Hubei Provincial Corps Hospital Chinese People's Armed Police Forces, Wuhan, Hubei, China. ${ }^{4}$ Department of Radiology, Affiliated Hospital of North Sichuan Medical College, Nanchong, Sichuan, China. ${ }^{5}$ Department of Radiology, Chongqing Wuxi County People's Hospital, Chongqing, China. ${ }^{6}$ Department of Radiology, Dianjiang People's Hospital of Chongqing, Chongqing, China.

Received: 8 March 2020 Accepted: 28 March 2020

Published online: 06 April 2020

\section{References}

1. Zhu N, Zhang D, Wang W, Li X, Yang B, Song J, Zhao X, Huang B, Shi W, Lu R, et al. A Novel coronavirus from patients with pneumonia in China, 2019. New England Journal Med. 2020;382:727-33.

2. Gorbalenya AE, Baker SC, Baric RS, de Groot RJ, Drosten C, Gulyaeva AA, Haagmans BL, Lauber C, Leontovich AM, Neuman BW, et al. Severe acute respiratory syndrome-related coronavirus-the species and its virusesa statement of the Coronavirus Study Group. BioRxiv. 2020 https://doi.org/10.1101/2020.02.07.937862

3. Guan WJ, Ni ZY, Hu Y, Liang WH, Ou CQ, He JX, Liu L, Shan H, Lei CL, Hui DS, et al. Clinical characteristics of 2019 novel coronavirus infection in China. MedRxiv. 2020. https://doi.org/10.1101/2020.02.06.20020974.

4. Radiological Diagnosis of New Coronavirus Infected Pneumonitis: Expert Recommendation from the Chinese Society of Radiology (First edition), Chin J Radiol 2020,54(00): E001-E001.

5. Fang $Y$, Zhang $H$, Xie J, Lin M, Ying L, Pang $P$, Ji W. Sensitivity of chest CT for COVID-19: comparison to RT-PCR. Radiology. 2020. https://doi. org/10.1148/radiol.2020200432

6. Lei H, Li Y, Xiao S, Lin CH, Norris SL, Wei D, Hu Z, Ji S. Routes of transmission of influenza A H1N1, SARS CoV, and norovirus in air cabin: comparative analyses. Indoor Air. 2018;28:394-403.

7. Otter JA, Donskey C, Yezli S, Douthwaite S, Goldenberg SD, Weber DJ. Transmission of SARS and MERS coronaviruses and influenza virus in healthcare settings: the possible role of dry surface contamination. J Hosp Infect. 2016;92:235-50.

8. Chen N, Zhou M, Dong X, Qu J, Gong F, Han Y, Qiu Y, Wang J, Liu Y, Wei $Y$, et al. Epidemiological and clinical characteristics of 99 cases of 2019 novel coronavirus pneumonia in Wuhan, China: a descriptive study. Lancet. 2020;395:507-13.

9. Liu WJ, Zhao M, Liu K, Xu K, Wong G, Tan W, Gao GF. T-cell immunity of SARS-COV: implications for vaccine development against MERS-CoV. Antiviral Res. 2017:137:82-92.

10. Ketai L, Paul NS, Wong KT. Radiology of severe acute respiratory syndrome (SARS): the emerging pathologic-radiologic correlates of an emerging disease. J Thorac Imaging. 2006;21:276-83.

11. Chong S, Kim TS, Cho EY. Herpes simplex virus pneumonia: high-resolution CT findings. Br J Radiol. 2010;83:585-9.

12. Hansell DM, Bankier AA, MacMahon H, McLoud TC, Muller NL, Remy J. Fleischner Society: glossary of terms for thoracic imaging. Radiology. 2008;246:697-722.

13. Xu Z, Shi L, Wang Y, Zhang J, Huang L, Zhang C, Liu S, Zhao P, Liu H, Zhu $L$, et al. Pathological findings of COVID-19 associated with acute respiratory distress syndrome. Lancet Respir Med. 2020. https://doi. org/10.1016/S2213-2600(20)30076-X.

14. Liu HF, Zhang DY, Yang Y, Long B, Yin L, Zhao M, Peng Y. Analysis of early chest high resolution $C T$ images of novel coronavirus pneumonia. Chin J Radiol. 2020;54(00):E007-E007.

15. Zhan X, Zhang L, Wang Z, Jin M, Liu M, Tong Z. Reversed halo sign: presents in different pulmonary diseases. PLOS ONE. 2015;10:e0128153-e0128153.

16. Chung M, Bernheim A, Mei X, Zhang N, Huang M, Zeng X, Cui J, Xu W, Yang Y, Fayad ZA, et al. CT Imaging Features of, Novel Coronavirus (2019-nCoV). Radiology. 2019;2020:200230-200230.

17. Liu J, Liu Y, Xiang P, Pu L, Xiong H, Li C, Zhang M, Tan J, Xu Y, Song $\mathrm{R}$, et al: Neutrophil-to-Lymphocyte Ratio Predicts Severe IIIness Patients with 2019 Novel Coronavirus in the Early Stage. medRxiv 2020:2020.2002.2010.20021584.

18. Guo L, Wei D, Zhang X, Wu Y, Li Q, Zhou M, Qu J. Clinical features predicting mortality risk in patients with viral pneumonia: the MuLBSTA score. Front Microbiol. 2019;10:2752-2752.

19. Wong KT, Antonio GE, Hui DSC, Lee N, Yuen EHY, Wu A, Leung CB, Rainer TH, Cameron P, Chung SSC, et al. Severe acute respiratory syndrome: radiographic appearances and pattern of progression in 138 patients. Radiology. 2003;228:401-6.

20. Das KM, Lee EY, Langer RD, Larsson SG. Middle East respiratory syndrome coronavirus: what does a radiologist need to know? AJR Am J Roentgenol. 2016;206:1193-201.

21. Ooi GC, Khong PL, Müller NL, Yiu WC, Zhou LJ, Ho JC, Lam B, Nicolaou $\mathrm{S}$, Tsang KW. Severe acute respiratory syndrome: temporal lung changes at thin-section CT in 30 patients. Radiology. 2004;230:836-44.

22. Das KM, Lee EY, Al Jawder SE, Enani MA, Singh R, Skakni L, AlNakshabandi N, AIDossari K, Larsson SG. Acute Middle East respiratory syndrome coronavirus: temporal lung changes observed on the chest radiographs of 55 patients. AJR Am J Roentgenol. 2015:205:267-74.

23. Koo HJ, Lim S, Choe J, Choi S-H, Sung H, Do K-H. Radiographic and CT features of viral pneumonia. Radiographics. 2018;38:719-39.

24. Gong $L$, Zhang C-L, Zhen Q. Analysis of clinical value of CT in the diagnosis of pediatric pneumonia and mycoplasma pneumonia. Exp Ther Med. 2016;11:1271-4.

25. Chen W, Xiong X, Xie B, Ou Y, Hou W, Du M, Chen Y, Chen K, Li J, Pei $L$, et al. Pulmonary invasive fungal disease and bacterial pneumonia: a comparative study with high-resolution CT. Am J Transl Res. 2019:11:4542-51

26. Wang D, Wu C, Gao J, Zhao S, Ma X, Wei B, Feng L, Wang Y, Xue X. Comparative study of primary pulmonary cryptococcosis with multiple nodules or masses by CT and pathology. Exp Therap Med. 2018;16:4437-44

\section{Publisher's Note}

Springer Nature remains neutral with regard to jurisdictional claims in published maps and institutional affiliations. 